\title{
Autonomic, EEG, and Behavioral Arousal Signs in a PVS Case After Zolpidem Intake
}

\author{
Calixto Machado, Mario Estévez, Jesús Pérez-Nellar, Joel Gutiérrez, Rafael Rodríguez, \\ Maylén Carballo, Mauricio Chinchilla, Andrés Machado, Liana Portela, \\ Maria C. García-Roca, Carlos Beltrán
}

Can J Neurol Sci. 2011; 38: 341-344

Several reports have been published over recent years about the paradoxical "arousing" effect of Zolpidem, a highly selective nonbenzodiazepine gamma aminobutyric acid (GABA) agonist acting on the [omega]-1 site of the GABA A receptor, in persistent vegetative state (PVS), in minimally conscious state (MCS) patients, ischemic stroke cases, after brain injury, and in patients suffering hypoxic encephalopathy. ${ }^{1}$

We describe here this paradoxical arousing effect in a PVS case assessing autonomic, electroencephalographic (EEG), and behavioral changes, after Zolpidem uptake. This case report is the first to show the importance of using heart rate variability (HRV) methodology and EEG to assess autonomic and brain functional changes in a PVS case, during this pharmacological intervention.

\section{METHODS}

We studied a 21-year-old female patient (YOR), who had suffered a stroke causing a top of the basilar artery syndrome and who had been in PVS for five years. Magnetic resonance imaging showed destruction of the rostral part of the pons, the mesencephalon, and both thalami. The patient showed circadian wakefulness, although she maintained longer periods of time with her eyes closed. With informed written consent of her parents, $10 \mathrm{mg}$ of Zolpidem were administered through a percutaneous endoscopic gastrostomy. A control healthy subject (DMC) matched for age and gender, without any history of autonomic or any other nervous system disorder, non-smoker, and who was not under any medication was also included. Zolpidem $10 \mathrm{mg}$ dissolved in $30 \mathrm{ml}$ saline solution was administered orally to subject DMC. This pharmacologic intervention was approved by the Institute of Neurology and Neurosurgery Ethics Committee, Havana, Cuba.

\section{Electroencephalographic studies}

Electroencephalographic records were obtained by the digital MEDICID-05 system (Neuronic S.A.), with the following technical parameters: gain 20,000, filters pass-band 0.1-30 Hz, $60 \mathrm{~Hz}$ "notch" filter, level of noise $2 \mu \mathrm{v}$ root mean square, sampling period of $5 \mathrm{~ms}$, and environmental temperature of approximately $23^{\circ} \mathrm{C}$. Nineteen monopolar leads were placed according to the International 10/20 System, using EEG Ag$\mathrm{AgCl}$ scalp electrodes, with linked ear lobes as a reference. Electrode-skin impedance was lower than $10 \mathrm{~K} \Omega$. Total time of recording was 75 minutes. The EEG records were independently assessed by two specialists of our staff for detailed clinical visual inspection and analysis. Free of artifacts EEG segments of 2.56 seconds (s) were selected for spectral analysis. Power spectral density (PSD) was estimated by averaging 25 segments (64 s) and results were compared with the established EEG quantitative norms available in this digital system considering age and gender variables. To study the EEG changes immediately before and after observed yawns, a program was developed in our Lab using MATLAB software version 7.10.0.499 (R2010a). The EEG segments of 40.96 seconds were selected, before and after every observed yawn. For every such segment the PSD was estimated using 8,192 samples. Spectral resolution was approximately $0.02415 \mathrm{~Hz}$ and EEG analysis was extended to absolute and normalized power indices in the subdelta band from 0.146 to 0.5 $\mathrm{Hz}^{2}$ The six EEG spectra obtained before and after every observed yawn were averaged to obtain the pre and postyawning averaged spectra. Usual spectral indices were also calculated.

\section{Autonomic studies}

Electrocardiogram was simultaneously digitally recorded with EEG and was exported offline to a custom-tailored software tool developed by our staff for visual inspection and detection of the "R" wave peaks. Accurate " $R$ " peak detections were visually controlled and properly corrected when it was necessary. The whole consecutive 80 minutes sequence of R-R intervals was submitted to a piecewise cubic Hermite interpolation process with a sampling frequency of approx-imately $6.83 \mathrm{~Hz}$, to transform the ordinal R-R sequence into a proper time series. Later, a dynamic time-frequency spectral analysis was applied. A time-window of five minutes duration was slid from the beginning to the end of the whole R-R sequence, overlapping in each displacement ten seconds of the R-R series. The power spectral density estimation and time-domain indices were

From the Institute of Neurology and Neurosurgery (CM, ME, JG, LP, MCGR, CB); Hermanos Ameijeras Hospital (JPN, MCh); International Center of Neurological Restoration (RR, MCa); University of Havana (AM), Faculty of Biology, Havana, Cuba.

Received June 25, 2010. Final Revisions Submitted October 19, 2010. Correspondence to: Calixto Machado, Institute of Neurology and Neurosurgery, Apartado Postal 4268, Ciudad de La Habana, 10400, Cuba. 


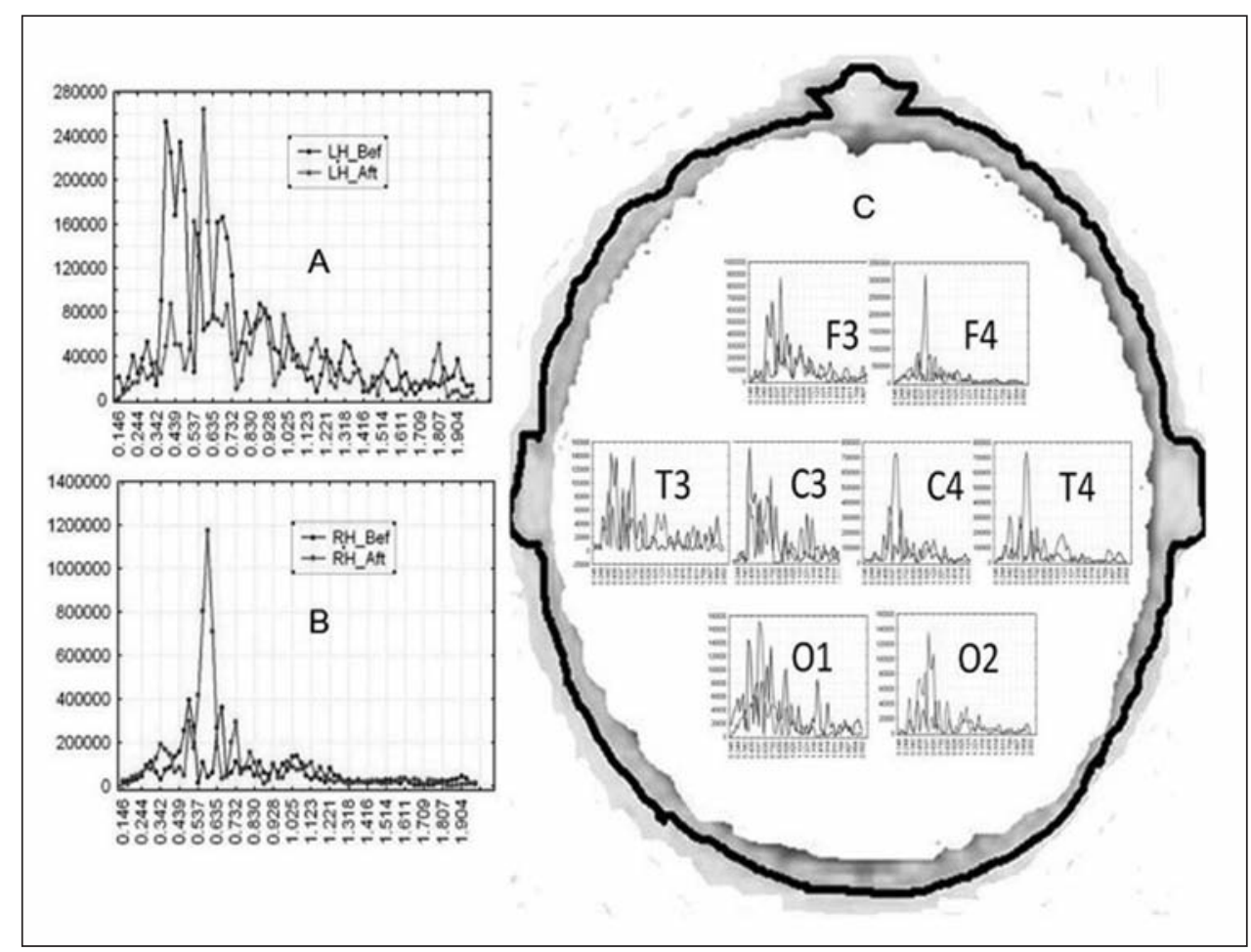

Figure 1: (A) EEG power averaged spectra calculated for F3 and F4. (B) Monopolar EEG leads in the range $0.146-2.0 \mathrm{~Hz}$, corresponding to 40.96 seconds immediately before (lighter line), and after (darker line) six yawns observed in patient YOR. The corresponding EEG power averaged spectra obtained in different monopolar EEG-leads before and after the six yawns are shown.

calculated for every window, using an FFT-based windowed periodogram method (Hann's window and 2048 samples). Spectral power was calculated for the very low frequency band in limits from 0.02 to $0.04 \mathrm{~Hz}$, the low frequency band from $0.04-0.15 \mathrm{~Hz}$, the high frequency band (HF) from $0.15-0.4 \mathrm{~Hz}$, and the total spectral power band (Tot) from 0.02-0.4 Hz. Zeta values were calculated for all time and spectral-domain indices, considering as control values those measures obtained during the pre-administration phase (20 min.) in patient YOR and in the control subject DMC. MATLAB software was also used for the time and frequency calculations of the R-R series. The control subject DMC was studied using the same procedure and experimental design used in patient YOR. Blood pressure was measured using the auscultatory method before Zolpidem administration in patient YOR and in subject DMC, and at minutes $10,20,30,40$, and 65 of the post-administration period.

For statistical assessment of observed differences between spectral indexes of EEG and HRV analysis we applied the Student "t" test for dependent samples, using the software Statistica 8.0 (StatSoft Inc 2007).

\section{RESULTS}

\section{Patient and control subject}

After the administration of Zolpidem, and for some 10-20 minutes, patient YOR began to open and close her eyes, until her eyes remained opened. Later, six spontaneous yawns appeared from minute 20 to 45 . Her eyes remained opened from Minute 25 to Minute 46. After this time, she closed her eyes, but continued with momentary eye opening and closing. During this last period neither yawns nor other evident arousal events in patient YOR were observed. For her parents, and for the members of our staff, these behavior signs were very unusual, because of the habitual sleep-like state otherwise characteristic of patient YOR. Subject DMC did not show any kind of symptoms during the recording session, except an obvious tendency to sleep evidenced by EEG monitoring.

\section{Electroencephalographic analysis}

Visual inspection of the EEG during the pre and postadministration periods of Zolpidem did not detect any obvious changes in patient YOR. The main feature in both experimental phases was the dominance of slow delta waves in all EEG leads. The relative PSD in the delta band comprised $89-96 \%$ of the whole EEG power density spectra in all EEG-leads, and the mean general EEG frequency from 0.5 to $30 \mathrm{~Hz}$ was $1.34 \mathrm{~Hz}$. Statistical comparisons of spectral indices in the frequency range from 0.5 to $30 \mathrm{~Hz}$, calculated in different stages after Zolpidem administration, did not reveal significant differences compared to basal control segments. Comparison of the delta spectral indices between the averaged pre-yawning EEG spectrogram with those obtained from the averaged spectrogram of the six post-yawning EEG segments, resulted in a significant increment of the delta power in the post-yawning segments, for the 


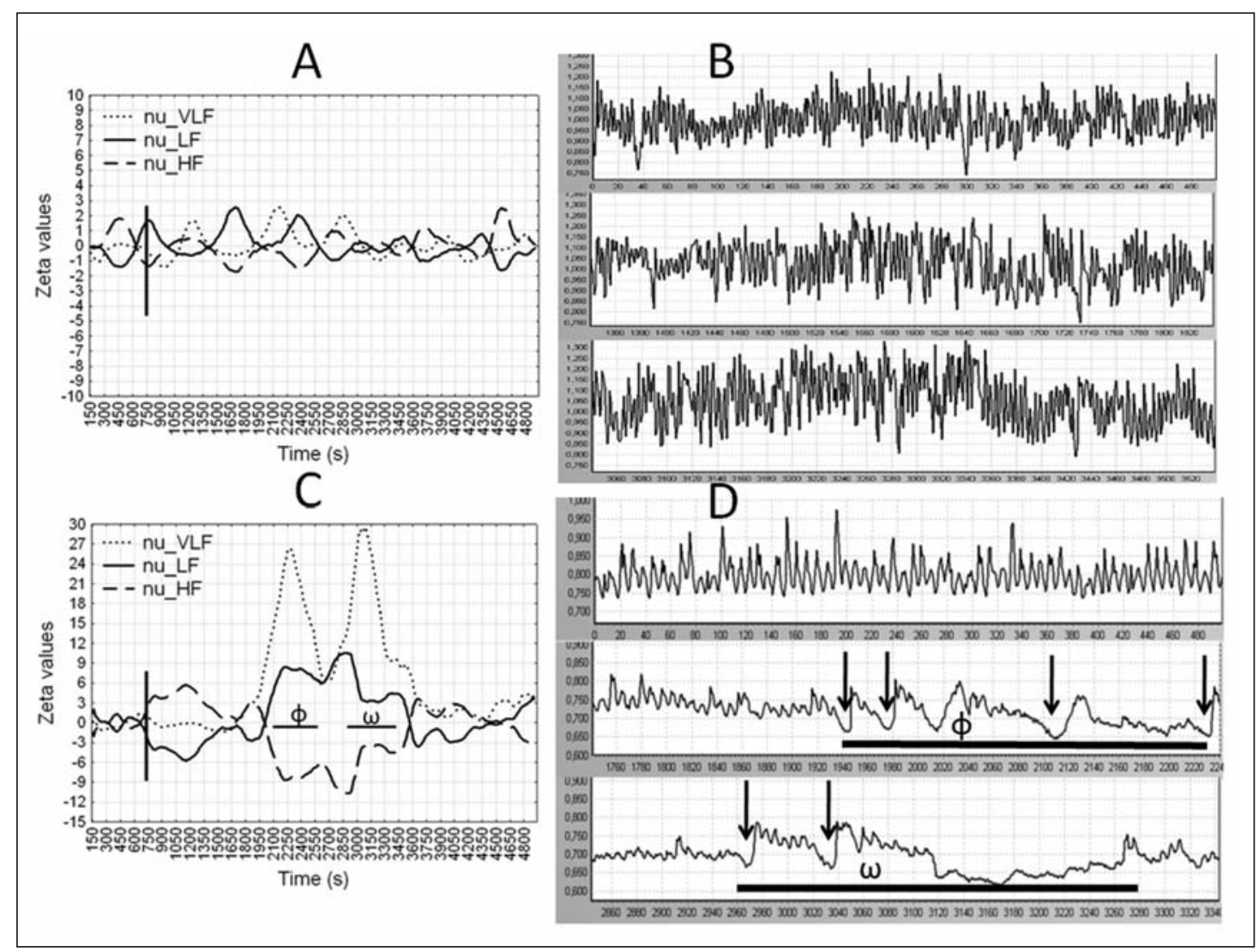

Figure 2: (A) In control subject, dynamic fluctuations of normalized units of the HRV power spectral density (PSD) in different spectral bands, expressed as zeta-values with respect to the control basal record (20 minutes), and later approximately one hour after Zolpidem, are shown. Vertical line identifies the moment of Zolpidem intake. In (B) three different sequential RR interval histograms from the control subject are presented. The upper histogram shows the first 500 RR intervals. The second one shows the RR intervals from 1360 to 1840, and the third one from 3040 to 3540 , both after Zolpidem intake. In $(C)$ the dynamic fluctuations in patient YOR during the control period (before the vertical line), and after Zolpidem intake, are presented. Values over 3 or under -3 zeta-values indicate a probability of $p<0.01$. In (D) the upper sequential histogram shows the first 500 RR intervals of patient YOR during the control basal pre-Zolpidem intake. The second and the third sequential histograms show the RR intervals from 1760 to 2240 and from the 3040 to the 3540, respectively. Vertical lines mark the moments associated with yawning manifestations in patient YOR. Greek letters $\varphi$ and $\omega$ identify the periods of yawnings and arousal signs in patient YOR.

frequency band pass of 0.5 to $30 \mathrm{~Hz}$. A similar comparison, exploring the frequency range from 0.146 to $30 \mathrm{~Hz}$ showed that in the pre-yawning spectrogram, absolute and normalized power indices in the subdelta band from 0.146 to $0.5 \mathrm{~Hz}$ were higher during the pre-yawning averaged spectrogram, and lower during the post-yawning spectrogram. A comparable analysis in the delta band confirmed increased values during the post-yawning averaged spectrogram, and diminished values in the pre-yawning averaged spectrogram, respectively. In Figure 1 the averaged spectrograms for some of the main EEG leads are presented. Considering that the spectral density values under $2 \mathrm{~Hz}$ were the most illustrative for this spectra analysis, in Figure 1 these results are confined to the 0.146 to $2.0 \mathrm{~Hz}$ frequency range.

In normal subject DMC EEG showed a progressive tendency to sleep reaching the non-REM Stages 1 and 2, approximately 20 minutes after Zolpidem intake, and continued fluctuating between these EEG sleep stages during the whole EEG record.

\section{Cardiovascular autonomic analysis}

Time and frequency domain indices calculated during the preadministration period in patient YOR did not show abnormal deviations from our laboratory norms. After the administration of Zolpidem the sequential histogram of R-R intervals showed a progressive reduction compared to the control pre-administration variability (Figure 2-D upper record). From minutes 20 to 32 after Zolpidem administration, and from minutes 37 to 45 , slow tachycardia waves were detected (Figure 2-D, middle and bottom histograms). Arrows indicate the moment when yawns were observed. Greek letters " $\varphi$ " and " $\omega$ " represent these two segments, when yawns were detected in YOR.

In Figure 2-C the dynamical fluctuation of different normalized bands spectral values, expressed as zeta values are shown. From minutes 3 to 10 after Zolpidem intake a significant 
$(\mathrm{p}<0.01)$ increment of nu_HF, and a significant reduction of nu_LF were observed. During the period when a yawning was observed, a highly significant increment of nu_VLF band was simultaneously detected. The nu_LF band was also significantly increased, while the nu_HF band showed a significant reduction during this period. After approximately 50 minutes normalized spectral fluctuations returned to pre-Zolpidem observed values.

In subject DMC the sequential histograms during basal record and after administration of Zolpidem $10 \mathrm{mg}$ dissolved in $30 \mathrm{ml}$ saline solution, did not change significantly (Figure 2-B), and the normalized values of the three spectral bands fluctuated from -2 to +2 SD regarding the mean control values.

In patient YOR, systolic blood pressure (BP) increased from $120 \mathrm{mmHg}$ to $138 \mathrm{mmHg}$, and the diastolic blood pressure (DP) only slightly augmented from 75 to 85 at 40 minutes after Zolpidem intake. In the control subject BP and DP values did not change, comparing pre and post Zolpidem $10 \mathrm{mg}$ administration periods.

\section{DISCUSSION}

Administration of Zolpidem in patient YOR was followed by significant behavioral signs of general arousal, such as periodic spontaneous movements of the eyes (openings and closings) and the presence of several yawns. Cardiovascular autonomic regulation showed initially, during the first ten minutes of the post-administration period, a significant parasympathetic predominance, followed by an ulterior ostensible significant sympathetic activation, which provoked a reduction of the overall HRV with peaks after Zolpidem, clearly coinciding with yawns and increased sympathetic predominance. Electroencephalography in our patient was dominated in all leads by very slow waves, with frequency in the subdelta band $(0.146$ $0.5 \mathrm{~Hz})$ and the lower delta frequency range $(0.5-2 \mathrm{~Hz})$. However, during the maximal tachycardia peaks, an electroencephalographic PSD displacement was observed from the subdelta range to a comparatively faster frequency range of delta waves. These EEG significant changes might be considered as correlates of brain arousal produced at the peak of sympathetic activation.

Maximal arousal of YOR was detected from 20 to 45 minutes after Zolpidem. Other reports also have found that this effect begins at this time. ${ }^{1}$ So far, no publication to our knowledge has reported yawning related to these paradoxical "awakenings" after Zolpidem administration.

Previous reports have not focused attention on autonomic cardiovascular regulation. ${ }^{1}$ Therefore, this case report is the first to show the importance of using HRV methodology in these patients. In a recent literature search (03-03-2010) on the international PubMed-NLM database, 394 publications were found under the keyword "Zolpidem". Of these, only eight included the keyword "heart rate", and only two were found using "heart rate variability" as keyword. In one of these papers, ${ }^{3}$ which studied physical fitness and altitude tolerance, the authors found that Zolpidem, used as a nocturnal hypnotic, produced a better sympathetic modulation to physical effort, shown by HRV analysis. Chen et al studied sleep-related vagotonic effect of zolpidem in rats, concluding that this drug induced a longer and deeper sleep, but it also led to an elevated cardiac vagal activity at a specific dose. ${ }^{4}$ Autonomic dynamics after Zolpidem in patient YOR, as described earlier, clearly showed periods of parasympathetic and of sympathetic cardiovascular predominance. The most interesting findings showed as sympathetic cardiovascular predominance coincided with yawns and behavioral signs of arousal.

Yawning is not a simple reflex, but it has a complex neural organization. It has been described in patients with various neurological disorders associated with disturbances of the ascending reticular activating system (ARAS), and it is considered an arousal response. The effect of different neurotransmitters that influence yawning, such as dopamine, serotonin, GABA, and others, has also been studied. ${ }^{5}$ In the bibliographic search in PubMed, previously described, we did not find any reference relating yawning and Zolpidem.

The EEG arousal activation observed in patient YOR, limited to changes from the subdelta band $(0.146-0.5 \mathrm{~Hz})$ to the lower delta frequency range $(0.5-2 \mathrm{~Hz})$, might be a consequence of the presence of destructive lesions located at the rostral part of the pons, mesencephalon and both thalami, preventing an adequate ARAS activating effect to the cerebral cortex.

Although this is report of a single case, our findings show a clear relationship between arousal behavioral signs and specific changes of the autonomic cardiovascular regulation, as well as the minimal but significant shift to faster frequencies of the EEG spectrum. This demonstrates the importance of assessing the socalled brain-heart connections to explain the paradoxical effect of Zolpidem in PVS and MCS patients, and merits further investigation.

\section{REFERENCES}

1. Clauss RP. Neurotransmitters in coma, vegetative and minimally conscious states, pharmacological interventions. Med Hypotheses. 2010; 75:287-90.

2. Rodin E, Modur P. Ictal intracranial infraslow EEG activity. Clin Neurophysiol. 2008; 119:2188-200.

3. Jouanin JC, Dussault C, Van BP, et al. Short half-life hypnotics preserve physical fitness and altitude tolerance during military mountainous training. Mil Med. 2009; 174:964-70.

4. Chen HY, Kuo TB, Shaw FZ, Lai CJ, Yang CC. Sleep-related vagotonic effect of zolpidem in rats. Psychopharmacology (Berl). 2005; 181:270-9.

5. Daquin G, Micallef J, Blin O. Yawning. Sleep Med Rev. 2001; 5: 299-312. 